\title{
Fuzzy Shrink Thresholding based Tea Leaf Image Enhancement using Wavelet Transform
}

\author{
Arunpriya C. \\ P.S.G.R Krishnammal College for Women \\ Coimbatore, India
}

\author{
Antony Selvadoss Thanamani \\ Nallamuthu Gounder Mahalingam College, \\ Pollachi, India
}

\begin{abstract}
In this paper a wavelet shrinkage algorithm based on fuzzy logic is proposed to improve the tea leaf image. The Tea Leaf images are normally changes to unclear images by the presence of noise, low or high dissimilarity both in the edge area and also in the image area. The Fuzzy shrink is used to enhance the image. In exacting, intra-scale dependency within wavelet coefficients is modeled using a fuzzy characteristic. This characteristic space distinguishes between significant coefficients, which depends on image discontinuity and noisy coefficients. This fuzzy characteristic is used for enhancing wavelet coefficients' information in the shrinkage step in this paper. Then a fuzzy membership function known as the spline-based curve is used to shrinks the wavelet coefficients based on the fuzzy characteristic. Here by using the interrelation between different channels as a fuzzy characteristic for improving the denoising performance compared to denoising each channel, separately. Examine the image denoising algorithm in the dual-tree discrete wavelet transform, which is the latest shiftable and customized version of discrete wavelet transform. Extensive comparisons with the high-tech image denoising algorithm indicate that the image denoising algorithm has a better performance in noise suppression and edge preservation as compared with the other methods. The spline based curve of a fuzzy membership function is more efficient one.
\end{abstract}

\section{General Terms}

Image Denoising, Wavelet Shrinkage Algorithm, Dual-Tree Discrete Wavelet Transform.

\section{Keywords}

Image Enhancement, Fuzzy Shrink, Tea Leaf Image, Denoising, Fuzzy, Wavelet Domain, Thresholding, and SSpline Curve.

\section{INTRODUCTION}

The application of digital image processing techniques for the problem of automatic leaf classification began two decades ago and it has since been proceeding in earnest. Into the industrial agriculture this type of technology found a number of its earlier applications to be extensively used. The Image sequence processing techniques are used to resolve the problems that occur in environmental biology. Plant plays a vital role for environment protection. Even though, the problem of plant damage becomes inferior in recent years. For this reason, many types of plants are at the risk of annihilation. To protect the plants and to catalogue various types, construction of automatic plant recognition system is a main step towards the conservation of earth's biosphere. There are several ways to distinguish a plant like flower, root, leaf, fruit etc. In recent times computer vision methodologies and pattern recognition techniques have been applied towards automated procedures of plant recognition $[1,2]$. Plant leaves are approximately two-dimensional in nature and the shape of plant leaf is one of the most important features for differentiating the various types of plants species. It helps in the development of an automatic method that can correctly categorize and recognize the leaf shapes of different species. These applications require high accuracy for the estimation of dynamic changes. Automatic classification and identification system for plant is essential and useful since it can facilitate the fast learning of plants [3, 4].

The Leaf images are normally changes to the blurred images by presence of noise, low or high contrast present in both the edge area and image area. Preprocessing is a technique in which an image includes removal of noise, edge or boundary enhancement, automatic edge detection, automatic contrast adjustment and the segmentation. As multiple noise damages the quality of nature of the images, improved enhancement technique is required for improving the contrast stretch in leaf images. Mostly the images in natural surface posses' low contrast as the features have a low range of reflectance in any waveband which affects the further development process of CAP-LR. CAP-LR generally includes the following steps: preprocessing, feature extraction, classification and recognition. Still, blurness and presence of unwanted noise on leaf images result in false classification. Thus image pre processing such as image enhancement techniques are highly needed to improve the quality of leaf image.

Image enhancement is basically improving the interpretability or discernment of information in images for human viewers and providing enhanced input for other automated image processing techniques $[5,6]$. During this process, one or more attributes of the image are altered. Selection of attributes and the way they are modified are specific to a given task. Image enhancement techniques are used to highlight certain features (i) rising the contrasts, (ii) altering the brightness level of an image so that the image looks better. This is essential for enhancing the Peak Signal-to-Noise Ratio (PSNR) and reducing the Mean Squared Error (MSE) for accurate identification. One of the most important objectives of leaf image enhancement is to enhance the contrast between regions of interest and the background. Also the medical images fluctuate extensively in terms of acquisition, noise characteristics and quality [3]. Thus, there is a requirement to process an image on the image basis. This motivates the design and construction of effective leaf image enhancement method using a new wavelet based fuzzy shrink and thresholding. The rest of this paper is organized as follows. The section 2 presents the related work, section 3 describes the proposed model, and some experimental results are illustrated in Section 4. At last, the conclusions are drawn in Section 5.

\section{RELATED WORK}

Xiao $\mathrm{Gu}$ et al., [7] proposed a novel approach for leaf recognition by means of the result of segmentation of leaf's skeleton based on the integration of Wavelet Transform (WT) 
and Gaussian interpolation. And then the classifiers, a nearest neighbor classifier (1-NN), a $\mathrm{k}$-nearest neighbor classifier (k$\mathrm{NN}$ ) and a radial basis probabilistic neural network (RBPNN) are employed, based on Run-length Features (RF) obtained from the skeleton to identify the leaves. Ultimately, the efficiency of this approach is illustrated by several experiments. The results reveal that the skeleton can be effectively extracted from the entire leaf, and the recognition rates can be significantly improved.

Wavelets are simply mathematical functions and these functions analyze data according to scale. They help in studying a signal at different resolutions or in different windows. Sendur and Selesnick used a bivariate shrinkage function, which models the arithmetical dependence sandwiched between a wavelet coefficient and its parent. It needs to estimate the marginal variance of the coefficient in a local neighborhood [8, 9];Pizurica and Philips used a probabilistic shrinkage function. Its core is estimating the probability that a given coefficient contains a significant noise-free component. Then the wavelet coefficient is multiplied with the probability [10]; Schulte et al. introduced a fuzzy version of probabilistic shrinkage method. Its core is shrinkage based on local mean of wavelet coefficients and some fuzzy rules [11]. Many other techniques have combined inter- and intra-scale dependencies. For example, denoising methods based on Gaussian Scale Mixture models, often employ the neighboring coefficients on the same and adjacent scales [12]. Local contextual HMT models have been developed, which captures both inter-scale and intra-scale dependent state $[13,14]$.

In image denoising, where a trade-off between noise suppression and the maintenance of actual image discontinuity must be made, solutions are required to detect important image details and accordingly adapt the degree of noise smoothing. In this paper, model the intra-scale dependency in wavelet transform domain as a fuzzy feature. Always postulate that noise is uncorrelated in the spatial domain; it is also uncorrelated in the wavelet domain. Based on this principle, we employ a fuzzy feature for single channel image denoising to enhance image information in wavelet sub-bands, and then using a fuzzy membership function to shrink wavelet coefficients, accordingly. This feature space distinguishes between important coefficients, which depends on image discontinuity and noisy coefficients. As for this reason and uncorrelated nature of noise, we use a new feature to extend our method for denoising multi channel images. DWT provides a good time frequency analysis of the signal, with a non-redundant signal representation and an optimal representation of singularities. Nevertheless, DWT suffers from five fundamental, intertwined shortcomings [15]: oscillations, aliasing, shift-variance, poor directionality, and absence of phase information. Shift invariance and directional selectivity are essential to the quality of wavelet based image denoising results. Because of the down-sampling operation in the DWT Filter Bank (FB), it is shift variance and will cause some visual artifacts (such as Gibbs phenomena) in thresholding- based denoising [16]. In addition, if the directional selectivity of a $\mathrm{FB}$ is defined as the ability to extract directional features into separate images, then the 2-D DWT has very poor directional selectivity because 2-D DWT has four sub-images. Many solutions to the shift-variance and lack of directionality of the DWT have been suggested in the literature. A simple approach to shift-variance is to remove the decimation blocks in the FB, so that there is no aliasing in the output sub-band signals. In this case, the sub-bands signals are perfectly shift-invariant (undecimated discrete wavelet transform). The new properties resulting from the use of this highly redundant transformation have been obtained at the expense of the loss of orthogonality, a considerably more intensive memory usage, and a higher computational cost than that of the original DWT. In this paper, examine the image denoising algorithm in the Dual-Tree DWT (DTDWT), which provides both shiftable sub-bands and good directional selectivity and low redundancy $[17,18]$ and [19].

\section{IMAGE ENHANCEMENT USING FUZZY SHRINK AND THRESHOLDING}

In this paper, proposed an efficient technique to enhance the tea leaf image using a new wavelet based fuzzy shrink and thresholding method. The following section discusses about the structural design and the use of fuzzy shrink in tea leaf image enhancement.

Fig 1 shows the system architecture of the proposed scheme. Initially, the Tea Leaf images are transformed to preprocessing process for preliminary enhancement. Then the output of preprocessing is given as input to the DT-DWT. In this transform, the preprocessing is not used as the initial step, although it is more efficient. Because it enhances the image contrast by transforming the pixel distribution as a result they can conform to uniform distribution.

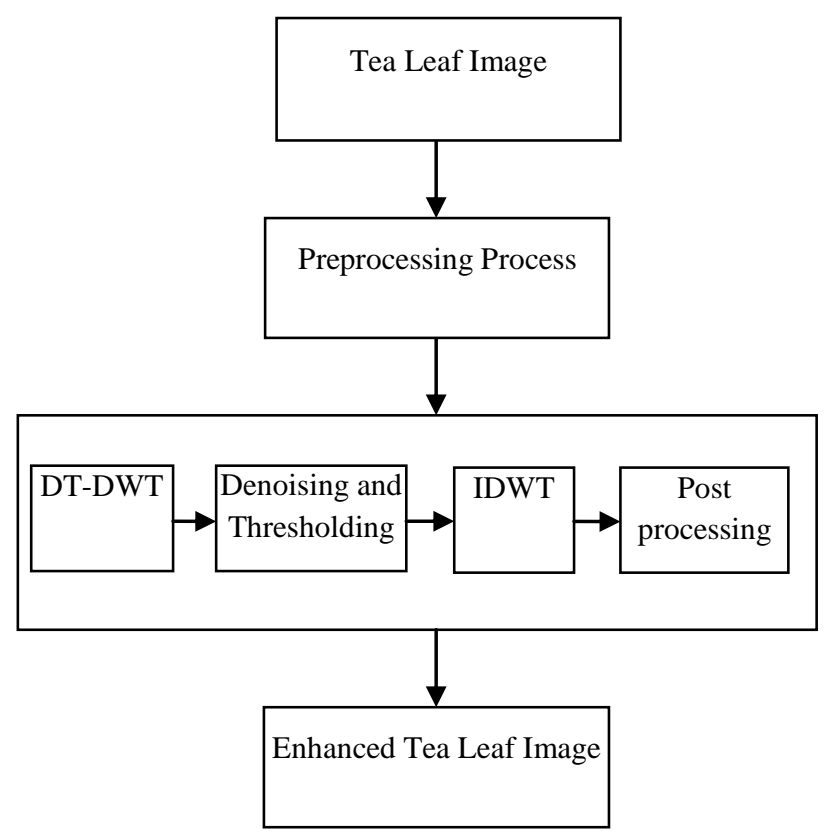
Fig 1: Diagram for the Proposed Tea Leaf Image
Enhancement

The input image which consist of noise is called noisy image is first transformed into wavelet domain. In the wavelet domain there are mainly three processes in which the image first compute the DUAL TREE discrete wavelet transform, pixel values are converted into wavelet coefficients then decomposed into six sub bands. After applying wavelet transform small coefficients are subjected by noise, while coefficients with a large absolute value carry more signal information than noise. Replacing the smallest, noisy coefficients by zero and a backwards wavelet transform on the result may lead to a reconstruction with the essential signal 
characteristics and with less noise. For thresholding three observations and assumptions:

1. The decorrelating property of a wavelet trans-form creates a sparse signal: most untouched coefficients are zero or close to zero.

2. Noise is spread out equally over all coefficients.

3. The noise level is not too high, so that can recognize the signal and the signal wavelet coefficients.

So, choosing of threshold level is important task the coefficients having magnitude greater than threshold are considered as signal of interest and keep the same or modified according to type of threshold selected and other coefficients become zero. The image is reconstructed from the modified coefficients. This process is also known as the inverse discrete wavelet transforms (IDWT). Selection of threshold is an important point of interest.

Care should be taken so as to preserve the edges of the denoised image. There will be various methods for wavelet thresholding, which depend on the choice of a threshold value. To choose the threshold value using fuzzy shrink rule, finally all the images are summed to low sub-band at the next to finer scale. The process is repeated until the image is reconstructed, at last get enhanced leaf image.

\subsection{Denoising and Thresholding}

Here the denoising is done through Fuzzy shrinkage rule. In image denoising, where a trade-off between noise suppression and the maintenance of actual image discontinuity must be made, solutions are required to detect important image details and accordingly adapt the degree of noise smoothing. With respect to this principle, use a fuzzy feature for single channel image denoising to enhance image information in wavelet sub-bands and then using a fuzzy membership function to shrink wavelet coefficients, accordingly.

\subsection{Fuzzy Feature}

Here, want to give large weights to neighboring coefficients with similar magnitude, and a small weight to neighboring coefficients with dissimilar magnitude. The larger coefficients are produced by noise which are always isolated or unconnected, but edge coefficients are clustered and persistent. It is well known that the more adjacent points are more similar in magnitude. So use a fuzzy function $\mathrm{m}(\mathrm{l}, \mathrm{k})$ of magnitude similarity and a fuzzy function $s(\mathrm{l}, \mathrm{k})$ of spatial similarity, which is defined as:

$$
\begin{aligned}
& \mathrm{m}(\mathrm{l}, \mathrm{k})=\exp \left(-\left(\frac{\mathrm{Y}_{\mathrm{s}, \mathrm{d}}(\mathrm{i}, \mathrm{j})-\mathrm{Y}_{\mathrm{s}, \mathrm{d}}(\mathrm{i}+\mathrm{l}, \mathrm{j}+\mathrm{k})}{\mathrm{Thr}}\right)^{2}\right) \\
& \mathrm{s}(\mathrm{l}, \mathrm{k})=\exp \left(-\left(\frac{\mathrm{l}^{2}+\mathrm{k}^{2}}{\mathrm{~N}}\right)\right)
\end{aligned}
$$

where $Y_{s, d}(i, j)$ and $Y_{s, d}(i+l, j+k)$ are central coefficient and neighbor coefficients in the wavelet sub-bands, respectively. Thr $=\mathrm{c} \times \widehat{\sigma}_{\mathrm{n}}, 3 \leq \mathrm{c} \leq 4, \widehat{\sigma}_{\mathrm{n}}$ is estimated noise variance, and $\mathrm{N}$ is the number of coefficients in the local window $k \in[-\mathrm{K} \ldots \mathrm{K}]$, and $\mathrm{l} \in[-\mathrm{L} \ldots \mathrm{L}]$.

According the two fuzzy functions, can get adaptive weight $\mathrm{w}(\mathrm{l}, \mathrm{k})$ for each neighboring coefficient:

$$
\mathrm{w}(\mathrm{l}, \mathrm{k})=\mathrm{m}(\mathrm{l}, \mathrm{k}) \times \mathrm{s}(\mathrm{l}, \mathrm{k})
$$

Using the adaptive weightsw $(\mathrm{l}, \mathrm{k})$, obtain the fuzzy feature for each coefficient in the wavelet sub-bands as follows:

$$
f(i, j)=\frac{\sum_{I=-L}^{L} \sum_{\mathrm{K}=-\mathrm{K}}^{\mathrm{K}} \mathrm{W}(\mathrm{l}, \mathrm{k}) \times\left|\mathrm{Y}_{\mathrm{s}, \mathrm{d}}(\mathrm{i}+\mathrm{l}, \mathrm{j}+\mathrm{k})\right|}{\sum_{\mathrm{I}=-\mathrm{L}}^{\mathrm{L}} \sum_{\mathrm{K}=-\mathrm{K}}^{\mathrm{K}} \mathrm{W}(\mathrm{l}, \mathrm{k})}
$$

\subsection{Fuzzy Shrinkage Rule}

The second step is want to give large weights to neighboring coefficients with similar magnitude, and a small weight to neighboring coefficients with dissimilar magnitude. The next step in the wavelet denoising procedure usually consists of shrinking the wavelet coefficients: the coefficients that contain primarily noise should be reduced to tiny values, while the ones containing a significant noise-free component should be reduced less. Here, use a fuzzy rule based on the fuzzy feature for shrinking the wavelet coefficients. Fuzzy logic was proposed by Zadeh [20], and has application in a large number of fields. The fuzzy sets and fuzzy rules form the knowledge base of a fuzzy rule-based reasoning system. Fuzzy rules are linguistic IF-THEN constructions that have the general form "IF A THEN B", where A and B are (collections of) propositions containing linguistic variables. The variable $\mathrm{A}$ is called the premise or antecedent and B is the consequence of the rule [21]. After finding the fuzzy feature, will form Linguistic IF-THEN rules for shrinking wavelet coefficients as follows:

IF the fuzzy feature $f(i, j)$ is large THEN shrinkage of wavelet coefficients $Y_{s, d}(i, j)$ is small.

In fact, the fuzzy feature indicates how coefficients in the noisy sub-band should be shrunk. Fuzzy membership function (MF) is a curve that defines how each point in the input space is mapped to a membership value (or degree of membership) between 0 and 1.MF is often given the designation of $\mu$. The input space is sometimes referred to as the universe of discourse, a fancy name for a simple concept. The only condition a membership function must really satisfy is that it must vary between 0 and 1 . The function itself can be arbitrary curves whose shape can be define as a function that suits us from the point of view of simplicity, convenience, speed and efficiency. MF is built from several basic functions such as: piece-wise linear functions, the sigmoid curve, quadratic curve, the Gaussian distribution function and cubic polynomial curves.

A cubic B-spline is a piecewise cubic polynomial that is twice continuously differentiable. This B-spline using here is the second order B-spline membership function and the general equation for unity is termed as

$$
\sum_{i=-m}^{N} B_{i, m}(x)=1, \quad x_{0} \leq x \leq x_{n}
$$

where $\mathrm{m}=0,1,2,3 \ldots$

Generally, the B-spline basis functions with order higher than 1 are not a normal fuzzy set, in the other words, the maximum value of the B-spline membership function does not reach 1 . Usually fuzzy systems theory requires that the membership functions are normal, this can be resolved by multiplying the $\mathrm{B}$-spline basis functions by a positive number so that its maximum value is 1 . In this paper using B-spline curve to get a smooth joint need to control the position and the curvature at the end points of the curve segments. These curves are highly flexible in nature than the $\mathrm{S}$ curves.

Here, the B-spline curve is using in which is a mapping on the vector $\mathrm{x}$, and is named because of its $\mathrm{B}$ - shape. The parameters $\mathrm{T} 1$ and $\mathrm{T} 2$ locate the extremes of the sloped portion of the curve as given by: 


$$
\mu(\mathrm{x})=\left\{\begin{array}{cr}
0 & \mathrm{x} \leq \mathrm{T}_{1} \\
2 \mathrm{X}\left(\frac{\mathrm{x}-\mathrm{T}_{1}}{\mathrm{~T}_{2}-\mathrm{T}_{1}}\right)^{2} & \mathrm{~T}_{1} \leq \mathrm{x} \leq \frac{\mathrm{T}_{1+} \mathrm{T}_{2}}{2} \\
1-2\left(\mathrm{~T}_{2}-\frac{\mathrm{x}}{\mathrm{T}_{2}-\mathrm{T}_{1}}\right)^{2} & \frac{\mathrm{T}_{1}+\mathrm{T}_{2} \leq \mathrm{x} \leq \mathrm{T}_{2}}{2} \\
1 & \mathrm{x} \geq \mathrm{T}_{2}
\end{array}\right.
$$

Here, also drawn an $S$ shaped curve [21] by using the mapping on the vector $\mathrm{x}$, and is named because of its $\mathrm{S}$ shape. By using the above parameters of the $\mathrm{T} 1$ and $\mathrm{T} 2$ the spline based curve also drawn with the B-Spline curve.

Finally, the estimated noise-free signal is obtained using the following formula:

$$
\hat{\mathrm{x}}_{\mathrm{s}, \mathrm{d}}(\mathrm{i}, \mathrm{j})=\mu(\mathrm{f}(\mathrm{i}, \mathrm{j})) \times \mathrm{Y}_{\mathrm{s}, \mathrm{d}}(\mathrm{i}, \mathrm{j})
$$

For building fuzzy membership function, two thresholds (T1 and T2), must be determined. Hence, found out that T1 and $\mathrm{T} 2$ are related with the $\widehat{\sigma}_{\mathrm{n}}$ which is the estimated noise variance. In order to find these relations, we have done some experiments using test images. We found out that T1 and T2 have nonlinear relation with the $\widehat{\sigma}_{\mathrm{n}}$. For achieving the nonlinear relation, have to test the noise reduction algorithm with the different noisy images. In each different noise variance, the result obtained best values for $\mathrm{T} 1$ and $\mathrm{T} 2$.

$$
\begin{aligned}
& \mathrm{T}_{1}=\mathrm{K}_{1} \times \widehat{\sigma}_{\mathrm{n}} \\
& \mathrm{T}_{2}=\mathrm{K}_{2} \times \widehat{\sigma}_{\mathrm{n}}
\end{aligned}
$$

Where $\mathrm{k} 1$ and the $\mathrm{k} 2$ are the constant values.

$\widehat{\sigma}_{\mathrm{n}}$ is the noise variance using median estimator.

$$
\widehat{\sigma}_{\mathrm{n}}=\frac{1}{\mathrm{~N}-1} \sum_{\mathrm{i}=1}^{\mathrm{N}}\left(\mathrm{x}_{\mathrm{i}}-\overline{\mathrm{x}}\right)^{2}
$$

Where $x_{i}$ is the selected pixels, $\bar{x}$ is the centered pixels and the $\mathrm{N}$ is the order of samples using this noise variance of the image is calculated.

Indeed, this method is a simple fuzzy IFTHEN rule, which assigns smaller local window and smaller level of decomposition when the estimated noise variance is small, and vice versa then the denoising output of the image is given to the inverse of the DWT for the reconstruction of the image. Then the image is sent for post processing process.

\subsection{Post Processing}

Processing is usually result from a modification of the spatial correlation between wavelet coefficients (often caused by zeroing of small neighboring coefficients) or by using DWT. DWT is shift invariance and will cause some visual artifacts in thresholding based denoising. For this reason, the fuzzy filter is used on the results of the proposed fuzzy-shrink algorithm to reduce artifacts to some extent. First, use a window of size $(2 \mathrm{~L}+1) \times(2 \mathrm{~K}+1)$ centered at $(\mathrm{i}, \mathrm{j})$ to filter the current image pixel at position $(i, j)$. Next, the similarity of neighboring pixels to the center pixel is calculated using the equation(1). Adaptive weight $\mathrm{w}(\mathrm{l}, \mathrm{k})$ for each neighboring coefficient is calculated using the equation(3). Finally, the output of post-processing step is determined as follows:

$$
\widetilde{\mathrm{x}}(\mathrm{i}, \mathrm{j}, \mathrm{c})=\frac{\sum_{\mathrm{I}=-\mathrm{L}}^{\mathrm{L}} \sum_{\mathrm{K}=-\mathrm{K}}^{\mathrm{K}} \mathrm{w}(\mathrm{l}, \mathrm{k}) \times \hat{\mathrm{x}}(\mathrm{i}+1, \mathrm{j}+\mathrm{k}, \mathrm{c})}{\sum_{\mathrm{I}=-\mathrm{L}}^{\mathrm{L}} \sum_{\mathrm{k}=-\mathrm{k}}^{\mathrm{k}} \mathrm{w}(\mathrm{l}, \mathrm{k})}
$$

where $\hat{\mathrm{x}}$ is the denoised image, which can be obtained using proposed fuzzy-shrink algorithm. After the post processing process the enhanced leaf image is obtained as a result.

\section{EXPERIMENTAL RESULT}

The Experimental Result that can be obtained as shown in the figure. When the input image is the Original image is shown in the figure 2(a) is undergoing decomposition then the image undergo denoising process in which the noise is removed, then the image is sent to the wavelet domain for the reconstruction of the image, finally get the enhanced image as shown in the figure $2(\mathrm{~b})$.

Objectively measured the results by the peak signal-to-noise ratio (PSNR) in decibels $(\mathrm{Db})$ can be measured using:

$$
\text { PSNR }=10 \log _{10}\left(\frac{255^{2}}{\mathrm{MSE}}\right)
$$

where

$$
\text { MSE }=\frac{1}{M \times N} \sum_{i=1}^{M} \sum_{j=1}^{N}(\hat{x}(i, j)-x(i, j))^{2}
$$

where $x(i, j)$ is the original image, $\hat{x}(i, j)$ is the estimated noise-free signal, and $\mathrm{M} \times \mathrm{N}$ is the number of pixels.

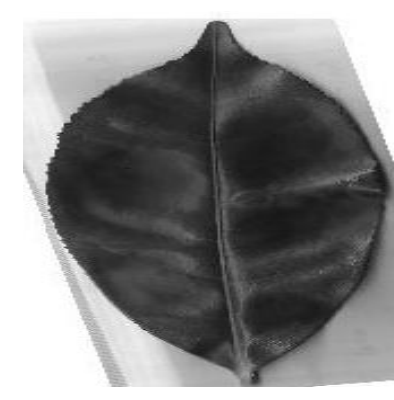

(a) Original Image

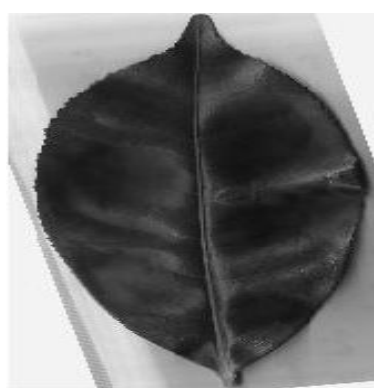

(b)Enhanced Image using B-Spline Membership Function

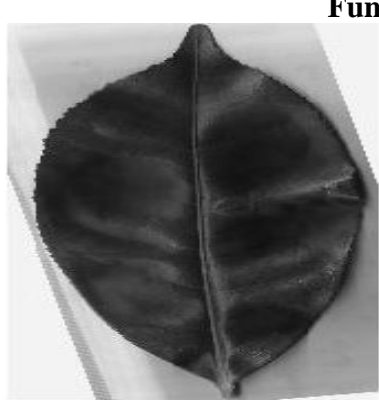

(c) Enhanced Image using Spline Based Membership Function

\section{Fig. 2: Resultant Image}

From the above Fig 2 came to know that the fuzzy membership function of spline based curve is more efficient then the B- Spline fuzzy membership function and also comparing with other methods.

The table 1 shows the comparison of PSNR and MSE values for various denoising methods to identify the tea leaf image. 
Table 1: Comparison of PSNR and MSE Value using Various Denoising Methods for Leaf Image Identification

\begin{tabular}{|c|c|c|}
\hline Various Transform & $\begin{array}{c}\text { PSNR Values in } \\
\text { Db }\end{array}$ & MSE Values \\
\hline Wavelet Method & 17.239 & 70.89 \\
\hline Curvelet Method & 27.239 & 53.28 \\
\hline Contourlet Method & 39.202 & 33.52 \\
\hline $\begin{array}{c}\text { Nonsubsampled } \\
\text { Contourlet Method }\end{array}$ & 45.039 & 11.01 \\
\hline $\begin{array}{c}\text { Fuzzy shrinkage } \\
\text { with B spline curve }\end{array}$ & 46.63 & 1.412 \\
\hline Spline -based Curve & 46.97 & 1.154 \\
\hline
\end{tabular}

From the table 1, shows the values of PSNR and MSE values of the various transforms. It is clear from the table that the proposed method is reliable and accurate one for image enhancement.

\section{CONCLUSION}

Leaf is one of the important organs of the plant. Leaf images are very noisy, low-contrast, blur and fuzzy and hence the leaf images are enhanced for accuracy. Processing these images require high computational capabilities. In this paper, the tea leaf images are enhanced using a new wavelet based fuzzy shrinkage rule and thresholding. In Wavelet thresholding, noise is eliminated by removing coefficient relative to some threshold. In the proposed method, soft thresholding is used. It is found from the experiments that the image enhancement method using fuzzy shrinkage is more efficient while comparing with other methods and it is useful in capturing relevant tealeaf information since its PSNR value is high with very low MSE value. In addition, the visual quality of the proposed denoised images exhibits the fewest number of artifacts and most of the edges is preserved. As a future work, the ability to incorporate more information is improved by adding other fuzzy rules to enhance the noise reduction performance.

\section{ACKNOWLEDGEMENT}

The authors acknowledge Dr. P. Mohan Kumar (Director), Dr. R. Victor J. Ilango (Sr. Botanist) and Dr. R. Raj Kumar (Sr. Plant Physiologist), UPASI Tea Research Foundation, Valparai, India, for providing tea leaf samples and basic taxonomical details to carry out this research.

\section{REFERENCES}

[1] Zhixin Shi, Srirangaraj Setlur, Venu Govindaraju, 2008. Digital Enhancement of Palm Leaf Manuscript Images using Normalization Techniques. IEEE conference. Computer Vision. Graphics \& Image Processing.687692.

[2] Manpreet Kaur, Jasdeep Kaur, Jappreet Kaur, 2011. Survey of Contrast Enhancement Techniques based on Histogram Equalization. (IJACSA) International Journal of Advanced Computer Science and Applications.2(7), 138-141.

[3] David Menotti, Laurent Najman, Jacques Facon, and Arnaldo de A. Araujo, 2007. Multi-Histogram Equalization Methods for Contrast Enhancement and Brightness Preserving. IEEE Transactions on Consumer Electronics. 53(3),1186-1193.

[4] Raman Maini and Himanshu Aggarwal, 2010. A Comprehensive Review of Image Enhancement Techniques. Journal of Computing. 2(3), 8-13.
[5] Sepasian, M., Balachandran, W. and Mares, C., 2008. Image Enhancement for Fingerprint Minutiae-Based Algorithms Using CLAHE. Standard Deviation Analysis and Sliding Neighborhood. Proceedings of the World Congress on Engineering and Computer Science WCECS. October 22 - 24, San Francisco, USA.

[6] Sharmila, R., Uma, R., 2011. A New Approach To Image Contrast Enhancement using Weighted Threshold Histogram Equalization with Improved Switching Median Filter. International Journal Of Advanced Engineering Sciences and Technologies. 7(2), 206-211.

[7] Xiao Gu, Ji-Xiang Du and Xiao-Feng Wang, 2005. Leaf Recognition Based on the Combination of Wavelet Transform and Gaussian Interpolation. Advances in Intelligent Computing. 3644, 253-262.

[8] Mallat, S., 1989. A theory for multiresolution signal decomposition: the wavelet representation. IEEE Transactions on Pattern Anal. Mach. Intell. 11(7),674 693.

[9] Donoho, D.L., Johnstone, I.M., 1995. Adapting to unknown smoothness via wavelet shrinkage. J. Amer. Statist. Assoc. 90 (432) , 1200-1224.

[10] Pizurica, A., Philips, W., 2006. Estimating the probability of the presence of a signal of interest in multiresolution single- and multiband image denoising. IEEE Transactions on Image Processing. 654-665.

[11] Schulte, S., Huysmans, B., Pizurica, A., Kerrel, E.E., Philips, W., 2006.A New Fuzzy-based Wavelet Shrinkage Image Denoising Technique. Springer Verlag. 12-23.

[12] Portilla, J., Strela, V., Wainwright, M., Simoncelli, E., 2003. Image denoising using Gaussian scale mixtures in the wavelet domain. IEEE Transactions on Image Processing. 1338-1351.

[13] Fan, G., Xia, X., 2001. Image denoising using local contextual hidden markov model in the wavelet domain, IEEE Signal Process. Lett. 125-128.

[14] Fan, G., Xia, X., 2001. Improved hidden Markov models in the wavelet domain. IEEE Trans. Signal Process. 115120.

[15] Selesnick, I.W., Baraniuk, R.G. , Kingsbury, N.G., 2005. The dual-tree complex wavelet transform. IEEE Signal Process. Mag. 124-152.

[16] Zhang, L., Bao, P., 2003. Hybrid inter and intra-wavelet scale image restoration. Pattern Recog. 36,1737-1746.

[17] Kingsbury, N.G., 2001. Complex wavelets for shift invariant analysis and filtering of signals. J. Appl. Computat. Harmonic Anal. 10 (3) , 234-253.

[18] Kingsbury, N.G. , 2000. A dual-tree complex wavelet transform with improved orthogonality and symmetry properties. Proc. IEEE Conf. Image Process. 375-378.

[19] Selesnick, I.W., 2002. The design of approximate Hilbert transform pairs of wavelet bases. IEEE Trans. Signal Process. 50 (5) , 1144-1152.

[20] Zadeh, L., 1965. Fuzzy Sets. Inform. Contr. 8(3),338353.

[21] Jamal Saeedi, Mohammad Hassan Moradi , Karim Faez, 2010. A new wavelet-based fuzzy single and multichannel image denoising. Image and Vision Computing 28, 1611-1623. 